\title{
Octogenarian newly diagnosed multiple myeloma patients without geriatric impairments: the role of age $>80$ in the IMWG frailty score
}

\author{
Mattia D’Agostino', Alessandra Larocca @i]', Massimo Offidani², Anna Marina Liberati $\mathbb{1}^{3}$, Gianluca Gaidano ${ }^{4}$, \\ Maria Teresa Petrucci ${ }^{5}$, Daniele Derudas $\mathbb{B}^{6}$, Andrea Capra ${ }^{1}$, Renato Zambello $\mathbb{B}^{7}$, Nicola Cascavilla ${ }^{8}$, Paolo de Fabritiis ${ }^{9}$, \\ Vanessa Innao ${ }^{10}$, Francesca Bonello ${ }^{1}$, Francesca Patriarca ${ }^{11}$, Giulia Benevolo ${ }^{12}$, Nicola Giuliani $\mathbb{1}^{13}$, Gabriele Aitoro ${ }^{14}$, \\ Tommasina Guglielmelli15, Francesco Di Raimondo ${ }^{16}$, Paolo Corradini (iD ${ }^{17}$, Pellegrino Musto ${ }^{18,19}$, Roman Hájek (10 ${ }^{20,21}$, \\ Pieter Sonneveld ${ }^{22}$, Mario Boccadoro (1) ${ }^{1}$ and Sara Bringhen (1)
}

Dear Editor,

In transplant-ineligible patients with newly diagnosed multiple myeloma (NDMM), impaired organ function and reduced physiological reserves may lead to a frail phenotype limiting the safe use of drugs and worsening patient outcome $\mathrm{e}^{1,2}$.

In 2015, the International Myeloma Working Group (IMWG) has developed an index to identify frail patients based on age, Charlson Comorbidity Index (CCI), Activities of Daily Living (ADL), and Instrumental ADL (IADL) ${ }^{3}$. Briefly, patients are stratified according to an additive score (range $0-5)$ evaluating age $(\leq 75$ years $=0$ points, $76-80$ years $=1$ point, $>80$ years $=2$ points $), C C I(\leq 1=0$ points, $\geq 2=1$ point), $\mathrm{ADL}(>4=0$ points, $\leq 4=1$ point), and IADL ( $>5=0$ points, $\leq 5=1$ point). Patients are classified as "fit" if the additive score is 0 , "intermediate fit" if the additive score is 1 , and "frail" if the additive score is $\geq 2$. According to this score, patients aged $>80$ years are determined to be frail independently from the presence of geriatric impairments (defined as $\mathrm{CCI} \leq 1$ and/or $\mathrm{ADL}>4$ and/or IADL > 5).

Since age in itself does not necessarily define biological frailty, the aim of our analysis was to describe the outcome of NDMM patients aged $>80$ years without geriatric impairments.

Correspondence: Sara Bringhen (sarabringhen@yahoo.com)

${ }^{1}$ Myeloma Unit, Division of Hematology, University of Torino, Azienda Ospedaliero-Universitaria Città della Salute e della Scienza di Torino, Torino, Italy

${ }^{2}$ Clinica di Ematologia, AOU Ospedali Riuniti di Ancona, Ancona, Italy

Full list of author information is available at the end of the article
We analyzed the original cohort that was used to define the IMWG frailty score, consisting of 869 transplantineligible NDMM patients enrolled in three prospective trials (EMN01, 26866138-MMY2069, and IST-CAR-506) ${ }^{3-6}$.

Frail patients were divided into two groups: patients who were determined to be frail by age only (Frail_by_age, i.e., patients aged $>80$ years with $\mathrm{CCI} \leq 1$ and $\mathrm{ADL}>4$ and IADL $>5$ ) vs. patients who were determined to be frail for any other reason (Frail_by_other).

The median follow-up was 65 months. Fit and intermediate-fit patients were used as reference population (No_frail, $n=609,70 \%$ ).

Among frail patients $(n=260,30 \%)$, only 70 patients were Frail_by_age (8.1\%). The remaining 190 frail patients (21.9\%) showed alterations in CCI ( $\geq 2$ in $43 \%$ of cases), ADL ( $\leq 4$ in $47 \%$ of cases), or IADL ( $\leq 5$ in $58 \%$ of cases) scores and were classified as Frail_by_other. Baseline characteristics are shown in Table 1.

As expected, Frail_by_age patients were older (median age 83) than Frail_by_other (median age 78) and No_frail patients (median age 72; $p<0.001$ ).

At diagnosis, Frail_by_age patients compared to Frail_by_other patients showed a better Eastern Cooperative Oncology Group Performance Status (ECOG $\geq 2$ in $12 \%$ vs. $52 \%, p<0.001)$ and less advanced disease (International Staging System [ISS] stage I in $31 \%$ vs. $14 \%$, $p<0.001$ ), similarly to No_frail patients.

No differences in terms of trial distribution (EMN01 69\% vs. $63 \%, 26866138-$ MMY2069 $29 \%$ vs. $33 \%$, IST-CAR-506 $4 \%$ vs. $3 \%$ ) and treatment received (Table S1) were found between Frail_by_age and Frail_by_other patients. 
Table 1 Baseline characteristics of Frail_by_age, Frail_by_other, and No_frail patients.

\begin{tabular}{|c|c|c|c|c|}
\hline Characteristics & Frail_by_age $N=70$ & Frail_by_other $N=190$ & No_frail $N=609$ & $p$-value* \\
\hline Median age (range) & $83(81-89)$ & 78 (67-91) & $72(50-80)$ & $<0.001$ \\
\hline Age, $n(\%)$ & & & & $<0.001$ \\
\hline$\leq 75$ & 0 & $46(24)$ & 477 (78) & \\
\hline $76-80$ & 0 & $96(51)$ & $132(22)$ & \\
\hline$>80$ & $70(100)$ & $48(25)$ & 0 & \\
\hline $\mathrm{CCl}, n(\%)$ & & & & $<0.001$ \\
\hline$\leq 1$ & $70(100)$ & $108(57)$ & $547(90)$ & \\
\hline$\geq 2$ & 0 & $82(43)$ & $62(10)$ & \\
\hline ADL, $n(\%)$ & & & & $<0.001$ \\
\hline$>4$ & $70(100)$ & $101(53)$ & $579(95)$ & \\
\hline$\leq 4$ & 0 & $89(47)$ & $30(5)$ & \\
\hline IADL, $n(\%)$ & & & & $<0.001$ \\
\hline$>5$ & $70(100)$ & $79(42)$ & $564(93)$ & \\
\hline$\leq 5$ & 0 & $111(58)$ & $45(7)$ & \\
\hline ECOG, $n(\%)$ & & & & $<0.001$ \\
\hline$\leq 1$ & $60(88)$ & $89(48)$ & $507(87)$ & \\
\hline$\geq 2$ & $8(12)$ & $95(52)$ & $77(13)$ & \\
\hline Missing & 2 & 6 & 25 & \\
\hline ISS, $n(\%)$ & & & & $<0.001$ \\
\hline I & $22(31)$ & $26(14)$ & $191(31)$ & \\
\hline$\|$ & $25(36)$ & $88(46)$ & $248(41)$ & \\
\hline III & $23(33)$ & $76(40)$ & $170(28)$ & \\
\hline FISH risk, $n(\%)$ & & & & 0.087 \\
\hline Standard & $38(64)$ & $117(71)$ & $368(76)$ & \\
\hline $\mathrm{High}^{* *}$ & $21(36)$ & $48(29)$ & $115(24)$ & \\
\hline Missing & 11 & 25 & 126 & \\
\hline
\end{tabular}

CCI Charlson Comorbidity Index, ADL Activities of Daily Living, IADL Instrumental Activities of Daily Living, ECOG Eastern Cooperative Oncology Group Performance Status, ISS International Staging System stage, FISH fluorescence in situ hybridization.

${ }^{*}$ Chi-squared test, Kruskal-Wallis test, or Fisher's exact test as appropriate.

**Highrisk by FISH defined by the presence of $t(4 ; 14)$ and/or $t(14 ; 16)$ and/or del17p13.

In a multivariate Cox model adjusted for ISS, cytogenetics, and treatment protocol, a worse overall survival (OS) was observed in both Frail_by_age (HR 1.51, $p=0.021)$ and Frail_by_other patients (HR $1.71, p<0.001$ ), as compared to No_frail patients (Fig. 1A). The median OS was 42.9 months in the Frail_by_age group, 41.6 months in the Frail_by_other group, and 76.5 months in the No_frail group.

Of note, no differences in terms of OS were found between Frail_by_age and Frail_by_other patients (HR $0.89, p=0.539$ ). Progression-free survival (PFS) and PFS2 data showed no significant differences between the two frail groups as well (Fig. S1A, B).

In the No_frail group, patients remained on study for a median time of 18.9 months, longer than patients in both the Frail_by_age (12.3 months) and Frail_by_other groups (12.4 months; Fig. S2).

Reasons for treatment discontinuation are reported in Table S2. The main reasons for discontinuation were adverse event $(60 \%)$ in the Frail_by_age group, adverse event (46\%) and disease progression (48\%) in the Frail_by_other group, and disease progression $(51 \%)$ in the No_frail group. A significantly higher risk of drug discontinuation for any cause, excluding progression and death, was reported in the Frail_by_age group (HR 2.34, $p<0.001$ ) and Frail_by_other group (HR 1.49, $p=0.003$ ), as compared to the No_frail group (Fig. 1B). Frail_by_age patients had a higher risk of drug discontinuation compared to Frail_by_other patients as well (HR 1.57, 


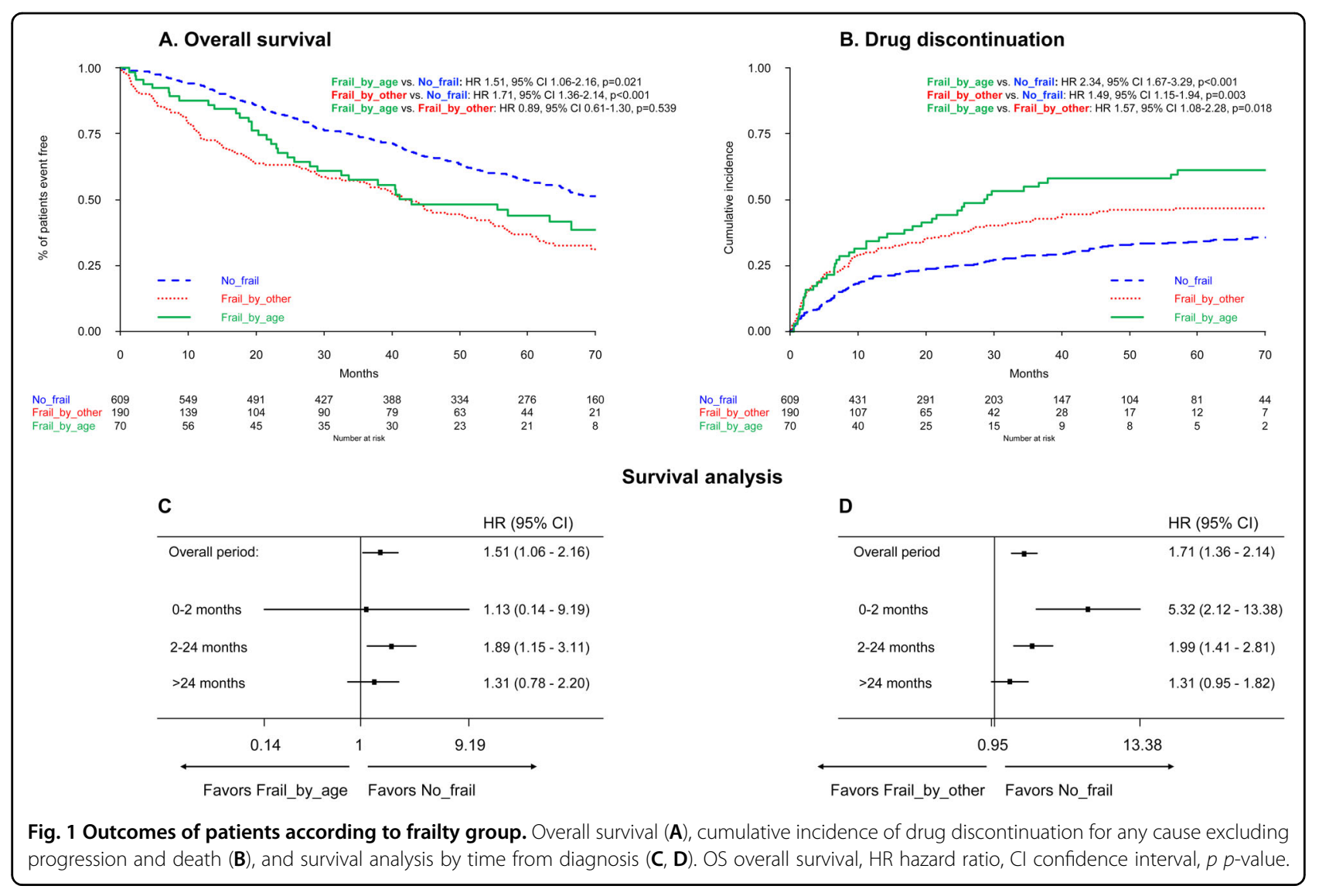

$p=0.018)$. Nevertheless, the cumulative incidence of grade $\geq 3$ non-hematologic and hematologic toxicities was not significantly different between Frail_by_age and Frail_by_other patients (Fig. S3A, B).

At the current follow-up, a second therapy was started in $61 \%$ vs. $66 \%$ vs. $72 \%$ of patients in Frail_by_age vs. Frail_by_other vs. No_frail groups, respectively. Among second therapies, low-dose conventional chemotherapy without novel agents was used in $37 \%$ vs. $24 \%$ vs. $7 \%$ of Frail_by_age vs. Frail_by_other vs. No_frail patients $(p<$ 0.001 ), suggesting that patients aged $>80$ years were more likely to receive a suboptimal therapy after the first line.

We next analyzed OS by dividing the time from diagnosis into three time frames to account for early deaths (0-2 months $)^{7}$, deaths within 2 years from diagnosis (2-24 months), and late deaths ( $>24$ months; Fig. 1C, D).

No significant differences in terms of early deaths were found between the Frail_by_age (HR 1.13, $p=0.909)$ and the No_frail groups, whereas a higher risk of early death was observed in the Frail_by_other group (HR 5.32, $p<0.001)$. Within the first 2 months, $21 / 869$ patients died overall $(2 \%)$, while this percentage was significantly higher in the Frail_by_other group (13/190, 7\%). The main cause of death in this time frame was death due to toxicity (62\%).
Between 2 and 24 months from diagnosis, both Frail_by_age (HR $1.89, p=0.012)$ and Frail_by_other (HR 1.99, $p<0.001)$ patients showed a significantly higher risk of death, as compared to No_frail patients. In this time frame, the main cause of death was progressive disease (65\%), followed by toxicity (24\%).

No differences in terms of late deaths were observed among the three groups. The main cause of late death was progressive disease (59\%), followed by toxicity (22\%).

To exclude an OS bias due to the older age of Frail_by_age patients, we explored the impact of geriatric impairments on patients aged $\leq 80$ years and $>80$ years (Fig. S4A, B). The presence of geriatric impairments significantly predicted a lower OS in the population aged $\leq 80$ years (HR 1.46, $p<0.001$ ), but not in the population aged $>80$ years (HR 1.09, $p=0.739$ ), thus supporting the hypothesis that octogenarian NDMM patients are frail independently from the presence of geriatric impairments.

To summarize our findings, octogenarian patients without geriatric impairments usually present with a low disease burden and a good performance status. However, the high rate of drug discontinuations and the difficulty to deliver effective treatments after the first line of treatment may lead to the observed poor survival. 
To date, this patient population is rare, accounting for $<10 \%$ of NDMM patients in clinical trials. Nevertheless, the life expectancy and health conditions of the general population are improving ${ }^{8}$. Thus, in the near future, physicians are expected to face a growing percentage of octogenarian NDMM patients without geriatric impairments .

New treatments (e.g., naked monoclonal antibodies) that can be safely delivered continuously for a long period of time may be better tolerated and have a lower discontinuation risk, potentially improving the outcome of this patient population. Indeed, dedicated trials selectively enrolling intermediate-fit and/or frail patients are beginning to emerge ${ }^{10,11}$. In a randomized phase III trial in intermediate-fit patients, 9 cycles of lenalidomidedexamethasone induction followed by low-dose lenalidomide maintenance without steroids produced similar outcomes compared to continuous lenalidomidedexamethasone (median PFS 20.2 vs. 18.3 months), thus showing that, in this patient subgroup, therapy could be de-intensified after induction without affecting patient outcome. Another trial enrolling both intermediate-fit and frail patients explored daratumumab-ixazomibdexamethasone induction followed by daratumumabixazomib maintenance. A total of $70 \%$ of intermediate-fit and $61 \%$ of frail patients completed induction treatment (9 months) and PFS rates were $78 \%$ and $61 \%$, respectively. The early death rate ( $\leq 3$ months after study entry) was higher in frail patients than in intermediate-fit patients (12\% vs. $0 \%)$.

Interestingly, in our work, we observed an excess of early toxic deaths ( $<2$ months from diagnosis) in patients who were frail due to geriatric impairments. This observation may support the exploration of dose-escalation strategies in the first months after diagnosis in frail NDMM patients presenting with geriatric impairments.

In conclusion, in this work, we showed that NDMM patients who were frail by age $>80$ years but who did not present with any geriatric impairments had a similar OS compared to patients who were determined to be frail for any other reason. These data further support that NDMM patients aged $>80$ years should be classified as frail regardless of the presence/absence of any comorbidities and ADL/IADL limitations.

\footnotetext{
Acknowledgements

G.G. acknowledges the role of the AGING Project - Department of Excellence DIMET, University of Eastern Piedmont (Novara, Italy) in the acquisition and analysis of the data reported in this contribution. The authors wish to thank all the study participants and referring clinicians for their valuable contributions; the nurses Carina Pusceddu and Cristina M. Russo; the data managers Federica Leotta and Marta Santoro; and Ugo Panzani from the Torino site.
}

\section{Author details}

${ }^{1}$ Myeloma Unit, Division of Hematology, University of Torino, Azienda Ospedaliero-Universitaria Città della Salute e della Scienza di Torino, Torino, Italy. ${ }^{2}$ Clinica di Ematologia, AOU Ospedali Riuniti di Ancona, Ancona, Italy. ${ }^{3}$ Università degli Studi di Perugia, Azienda Ospedaliera Santa Maria, Terni, Italy.
${ }^{4}$ Division of Hematology, Department of Translational Medicine, University of Eastern Piedmont, 28100 Novara, Italy. ${ }^{5}$ Hematology, Department of

Translational and Precision Medicine, Azienda Ospedaliera Policlinico Umberto I, Sapienza University of Rome, Rome, Italy. ${ }^{6} \mathrm{SC}$ di Ematologia e CTMO,

Ospedale Oncologico di Riferimento Regionale "A. Businco", ARNAS "G. Brozu", Cagliari, Italy. ${ }^{7}$ Padova University School of Medicine, Hematology and Clinical Immunology, Padova, Italy. ${ }^{8}$ Division of Hematology, IRCCS "Casa Sollievo della Sofferenza" Hospital, San Giovanni Rotondo, Italy. ${ }^{9}$ Hematology, St. Eugenio Hospital ASL Roma 2, Tor Vergata University, Rome, Italy. ${ }^{10}$ Division of Hematology, Department of Human Pathology in Adulthood and Childhood, University of Messina, 98122 Messina, Italy. ${ }^{11}$ Clinica Ematologica e Unità di Terapie Cellulari, Azienda Sanitaria Universitaria Friuli Centrale (ASUFC), Dipartimento di Area Medica (DAME), Università di Udine, Udine, Italy. ${ }^{12} \mathrm{SC}$ Hematology, AO Città della Salute e della Scienza, Turin, Italy. ${ }^{13}$ Hematology, "Azienda Ospedaliero-Universitaria" and Department of Medicine and Surgery, University of Parma, Parma, Italy. ${ }^{14}$ Department of Medicine, Azienda Sanitaria Locale Torino 4, Cirié, Chivasso e Ivrea, Italy. ${ }^{15}$ Ematologia, Ospedale San Luigi Gonzaga, Orbassano, Italy. ${ }^{16}$ Division of Hematology, AOU Policlinico, University of Catania, Catania, Italy. ${ }^{17}$ Divisione di Ematologia, Fondazione IRCCS Istituto Nazionale dei Tumori di Milano, Università degli Studi di Milano, Milano, Italy. ${ }^{18}$ Department of Emergency and Organ Transplantation, "Aldo Moro" University School of Medicine, Bari, Italy. ${ }^{19}$ Unit of Hematology and Stem Cell Transplantation, AOUC Policlinico, Bari, Italy. ${ }^{20}$ Department of Haematooncology, University Hospital Ostrava, Ostrava, Czech Republic. ${ }^{21}$ Faculty of Medicine, University of Ostrava, Ostrava, Czech Republic.

${ }^{22}$ Department of Hematology, Erasmus MC Cancer Institute, Rotterdam, the Netherlands

\section{Author contributions}

M.D., A.L., M.B., and S.B. conceived and designed the work that led to the submission. All authors acquired the data and interpreted the results. M.D., A.L., and S.B. drafted the first version of the manuscript. A.C. performed the statistical analysis. All authors revised the manuscript and approved the final version. All authors agreed to be accountable for all aspects of the work in ensuring that questions related to the accuracy or integrity of any part of the work are appropriately investigated and resolved.

\section{Conflict of interest}

M.D. has received honoraria for lectures from Sanofi and GSK; has served on the advisory boards for GSK. A.L. has received honoraria from Amgen, Bristol-Myers Squibb, Celgene, Janssen, and GSK; has served on the advisory boards for BristolMyers Squibb, Celgene, Janssen, and Takeda. M.O. has received honoraria from Amgen, Bristol-Myers Squibb, Celgene, Janssen, GSK, Sanofi, and Takeda; has served on the advisory boards for Amgen, Bristol-Myers Squibb, Celgene, Janssen, GSK, Sanofi, and Takeda. A.M.L. has received personal fees from Incyte; has received research funding from Novartis, Janssen, AbbVie, Roche, Celgene, Amgen, Bristol-Myers Squibb, Takeda, Incyte, Pfizer, Beigene, Oncopeptides, Verastem, Karyopharm, Archigen, Biopharma, Debiopharm, Morphosys, Fibrogen, and Onconova. G.G. has served on the advisory boards for Janssen, AbbVie, and AstraZeneca; has served on the speaker's bureaus for Janssen and AbbVie. M.T.P. has received honoraria from and served on the advisory boards for Celgene, Janssen-Cilag, Amgen, Bristol-Myers Squibb, Takeda, Sanofi, and GSK. R.Z. has served on the advisory boards for Janssen and Celgene. F.P. has served on the advisory boards for Celgene and Janssen. G.B. has received honoraria from Novartis, Celgene Amgen, and Takeda. N.G. has received research grants from Celgene and Janssen Pharmaceutica; has received sponsorship for clinical studies from Janssen Pharmaceutica, Millennium Pharmaceuticals, and GSK; has served on the advisory boards for Celgene, Takeda, and Janssen Pharmaceutica; has received support for participating in meetings from Janssen Pharmaceutica, Celgene, and Bristol-Myers Squibb. F.D.R. has received honoraria from Celgene, Janssen, Amgen, and Takeda. P.C. has participated as a lecturer and/or has served on the advisory boards for AbbVie, ADC Therapeutics, Amgen, Celgene, Daiichi Sankyo, Gilead, Incyte, Janssen, Jazz Pharmaceuticals, Kite, Kyowa Kirin, Novartis, Roche, Sanofi, Servier, and Takeda. P.M. has received honoraria and/or served on the scientific advisory boards for Celgene, Janssen, Takeda, Bristol-Myers Squibb, Amgen, Novartis, Gilead, Jazz, Sanofi, AbbVie, and Glaxo-Smith-Kline. R.H. has had a consultant or advisory relationship with Janssen, Amgen, Celgene, AbbVie, BMS, Novartis, PharmaMar, and Takeda; has received honoraria from Janssen, Amgen, Celgene, BMS, PharmaMar, and Takeda; has received research funding from Janssen, Amgen, Celgene, BMS, Novartis, and Takeda. P.S. has served on the advisory boards for Amgen, Celgene, Genenta, Janssen, Seattle Genetics, Takeda, 
and Karyopharm. M.B. has received honoraria from Sanofi, Celgene, Amgen, Janssen, Novartis, Bristol-Myers Squibb, and AbbVie; has served on the advisory boards for Janssen and GSK; has received research funding from Sanofi, Celgene, Amgen, Janssen, Novartis, Bristol-Myers Squibb, and Mundipharma. S.B. has received honoraria from Celgene, Amgen, Janssen, and Bristol-Myers Squibb; has served on the advisory boards for Celgene, Amgen, Janssen, and Karyopharm; has received consultancy fees from Janssen and Takeda. The remaining authors declare no competing interests.

\section{Publisher's note}

Springer Nature remains neutral with regard to jurisdictional claims in published maps and institutional affiliations.

Supplementary information The online version contains supplementary material available at https://doi.org/10.1038/s41408-021-00464-w.

\section{Received: 11 January 2021 Revised: 15 March 2021 Accepted: 29 March} 2021

Published online: 12 April 2021

\section{References}

1. Larocca, A. et al. Patient-centered practice in elderly myeloma patients: an overview and consensus from the European Myeloma Network (EMN). Leukemia 32, 1697-1712 (2018).

2. Salvini, M., D’Agostino, M., Bonello, F., Boccadoro, M. \& Bringhen, S. Determining treatment intensity in elderly patients with multiple myeloma. Expert Rev. Anticancer Ther. 18, 917-930 (2018).
3. Palumbo, A. et al. Geriatric assessment predicts survival and toxicities in elderly myeloma patients: an International Myeloma Working Group report. Blood. 125, 2068-2074 (2015).

4. Bringhen, $\mathrm{S}$. et al. Lenalidomide-based induction and maintenance in elderly newly diagnosed multiple myeloma patients: updated results of the EMN01 randomized trial. Haematologica 105, 1937-1947 (2020).

5. Larocca, A. et al. A phase 2 study of three low-dose intensity subcutaneous bortezomib regimens in elderly frail patients with untreated multiple myeloma. Leukemia 30, 1320-1326 (2016).

6. Bringhen, S. et al. Carfilzomib, cyclophosphamide, and dexamethasone in patients with newly diagnosed multiple myeloma: a multicenter, phase 2 study. Blood. 124, 63-69 (2014).

7. Bringhen, S. et al. Early mortality in myeloma patients treated with firstgeneration novel agents thalidomide, lenalidomide, bortezomib at diagnosis: a pooled analysis. Crit. Rev. Oncol. Hematol. 130, 27-35 (2018).

8. United Nations - Department of Economic and Social Affairs - Popular Division. World Population Ageing 2019 (ST/ESA/SER.A/444) (United Nations, New York, 2020).

9. Mina, R., Bringhen, S., Wildes, T. M., Zweegman, S. \& Rosko, A. E. Approach to the Older Adult With Multiple Myeloma. Am. Soc. Clin. Oncol. Educ. B 39, 500-518 (2019).

10. Larocca, A. et al. Dose/Schedule-Adjusted Rd-R vs Continuous Rd for elderly, intermediate-fit, newly diagnosed multiple myeloma patients. Blood. https:// doi.org/10.1182/blood.2020009507. Online ahead of print (2021).

11. Stege, C. A. M. et al. Efficacy and Tolerability of Ixazomib, Daratumumab and Low Dose Dexamethasone (Ixa Dara dex) in Unfit and Frail Newly Diagnosed Multiple Myeloma (NDMM) Patients; Results of the Interim Efficacy Analysis of the Phase II HOVON 143 Study. Blood. Vol. 134, Abstract \#695 [ASH 2019 61st Meeting] (2019). 